\title{
A LITERATURE REVIEW ON SEMANTIC WEB - UNDERSTANDING THE PIONEERS' PERSPECTIVE
}

\author{
Salih Ismail ${ }^{1}$ and Talal Shaikh ${ }^{2}$ \\ ${ }^{1}$ Mathematical and Computer Sciences, Heriot Watt University, Dubai, UAE \\ si8@hw.ac.uk \\ ${ }^{2}$ Mathematical and Computer Sciences, Heriot Watt University, Dubai, UAE \\ t.a.g.shaikh@hw.ac.uk
}

\begin{abstract}
There are various definitions, view and explanations about Semantic Web, its usage and its underlying architecture. However, the various flavours of explanations seem to have swayed way off-topic to the real purpose of Semantic Web. In this paper, we try to review the literature of Semantic Web based on the original views of the pioneers of Semantic Web which includes, Sir Tim Berners-Lee, Dean Allemang, Ora Lassila and James Hendler. Understanding the vision of the pioneers of any technology is cornerstone to the development. We have broken down Semantic Web into two approaches which allows us to reason with why Semantic Web is not mainstream.
\end{abstract}

\section{KEYWORDS}

Semantic Web, Literature Review, Pioneers' Perspective

\section{INTRODUCTION}

This "The power of the Web is in its universality. Access by everyone regardless of disability is an essential aspect." - Sir Tim Berners-Lee (Inventor of the World Wide Web) [1]

The World Wide Web (WWW) was created by Sir Tim Berners-Lee with the vision of connecting people. It didn't take long for WWW to become a global phenomenon and become the backbone for communication on a global level. So much information has been uploaded on the Web that, information could be found just about anything on the Web. This phenomenon kept on growing and essentially the Web has become the brain of planet Earth. [2] There is approximately 100 petabytes of data available on the Internet. [3]

How can you find the right information about a particular topic from this vast ocean of data depends on who wants to find the right information, whether it is a human being or a machine. Search engines have become so important because it helps us retrieve various information about a particular topic. But human beings are capable to compare the different webpages and make an informed choice because, we understand the 'meaning of the information'. 
The existing Web does provide a fair degree of aid for machines to find information. But definitely that's not enough to find the right information. Currently the extent of the capability of machines are to find the information based on a keyword match and its variants. But machines for a fact do not understand the meaning of those keywords. Many Artificial Intelligence researchers believe that Machine Learning is the key to unlocking machine understanding. However, Machine Learning is hugely based on mathematical equations and statistical analysis. It is computationally very costly even to perform the smallest of tasks, like identifying an image. [4]

"If I have a virtual personal assistant and have somebody who is helping me do my shopping, you are essentially selling to the machine or my agent. Suddenly, that means you need to be good at data. It means that you need to make sure that you have all your products and all the scripts are described in the data that the machine understands." - Sir Tim Berners-Lee [5]

Sir Tim Berners-Lee believes that this is only possible with the adoption of the 'Semantic Web'.

Semantic Web is the evolution of the WWW due to the enhancement in other parallel technologies like pervasive computing, sentient computing, internet of things, artificial intelligence etc. Semantic Web tries to classify the data based on different topics and assign meaning to it. This would in turn not only aid in better human understanding, but also in enhancing the understanding of the machines. Truly the power of WWW can become an extension of the human mind's capacity at this point.

Even though the importance of Semantic Web has been stressed upon by various technology futurists and other respected personalities within the technology world, nothing disruptive has been happening towards this area. [6] [7]

There are tasks and processes which human beings are far superior to computer and vice-versa. Semantic Web would enable machines and humans to cooperatively perform tasks, which would utilize the strength of both realms and provide with a better result.

If machines are able to find commonalities and anomalies among various ontologies, they would be able to create a knowledge base that would really enhance the understanding of the machines. And if machines understand better, then humans would be able to delegate a lot of tasks that a machine could do better and faster. The real essence of 'Co-computing' would become a reality by the use Semantic Web.

\section{STRUCTURE OF THE REPORT}

The introduction section above, threw light on setting the tone at which the pioneers looked upon at Semantic Web and how do this technology fit in to the realm of the machines.

The section below, deals with the Literature Review, where we explain the basic concepts and terminologies of Semantic Web briefly as per the descriptions and explanations of the pioneers. Finally, we explain few criticisms that Semantic Web faces and what are the replies provided by the creators of Semantic Web.

Finally, we conclude the paper by summarizing the essence of the vision of the creators of Semantic Web and what would be a good start to refresh the perspective about Semantic Web. 


\section{RELATED WORKS}

There is a lot of literature review that has been done on Semantic Web, but very few work has been done reiterating the idea behind Semantic Web from the pioneers' perspective. The real essence of where and why Semantic Web should be used has really deviated. The introduction was a snapshot of the vision of what Semantic Web should be doing. On these lines, Janev and Vrane has spoken about few concepts of what Semantic Web can do and also done a survey on the existing tools and languages available to achieve it [28]. But they are focusing on the constraints of Semantic Web in general and not particular to the bigger role that Semantic Web should be playing.

Guns Raf has done another analysis on tracing back the origins of Semantic Web [29]. He tried to counter debate the criticism of Semantic Web just being Web. They have traced back Semantic Web origins back to early concepts of Artificial Intelligence. This would prove to be in alignment with the concepts of how Semantic Web can be used according to Sir Tim Berners-Lee.

Benslimane et al. discuss the importance of how Semantic Web can become important for machines if there is proper method to structure existing data into Semantic format using RDF and OWL [30]. This is again an outcome of Semantic Web's real use.

We will try to explain the literature that is available to understand Semantic Web in its real essence in the following section.

\section{LITERATURE REVIEW}

\subsection{From Web to Semantic Web}

Most people thought WWW wouldn't become successful as there a lot of problems like, who will upload the data, who will manage it, who will fix the issues etc. But the widespread adoption of WWW has been on a planetary level and just about everything has a webpage for it.

The Web infrastructure currently is a distributed network of interlinked webpages with Unique Resource Locators. This helps to categorize webpages of a particular niche and identify them. The idea of Semantic Web is to push the very same infrastructure, where the linking of resources is on the data level. Semantic Web is based on the idea of Smart Data [9].
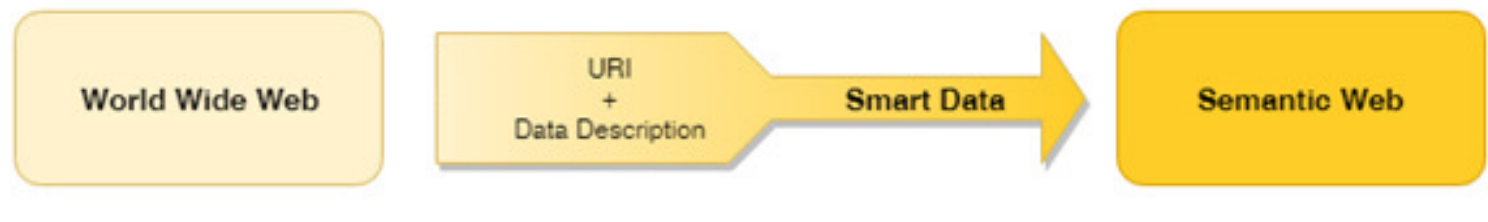

Figure 1: From Web to Semantic Web.

Smart Data is interlinked data that allows not only humans to use the information, but machines too. Even if each entity of the data is held by individual organization, since they are all interlinked, it could make more meaning [10]. Sir Tim Berners-Lee believed that when interlinked data could also have the property of self-description, it would lead to Semantic Web. 
Some of the features of Semantic Web are to be compared with how the Web itself was developed to understand it better:

- The voice - WWW took off to a flying start because anyone was able to say anything about any topic (AAA slogan). There could be hundreds of opinions about a particular topic and it is up to the reader to make a decision. This is one of the striking phenomenon that led to WWW becoming a global endeavor. But this also resulted in the Web becoming a place full of information but hard to find out the "right information". Semantic Web needs to allow the same heterogeneity of data, but at the same time have a small model to start off the discussion on any topic. For this purpose, RDF was created which helps to link data [9].

- Content creators - The most resounding question to the proposition of the Web was "who is going to create pages?" The answer to this question was that "everyone would create content". It proved to be true against the speculation of the skeptics, and to even the proponents of the Web. The same concept needs to be borrowed for Semantic Web.

The web already proved to us that content wouldn't be a problem and it will eventually be populated. The same goes for Semantic Web. The Web grew because of the 'network effect'. Crowd sourced contents like Wikipedia and IMDB made their entry and grew into massive sources of information [9].

- The users - The Web was meant for humans to share information with one another. Semantic Web has another user - "Machines". One of the major reasons as to why the importance of Semantic Web is increasing is the evolution of Pervasive Computing and Internet of Things. The estimated increase of the number of devices are exponential [7]. This means only one thing - "More Data". If all this data would have been linked to each other, the potential of this data would be massive [9].

\subsection{Semantic Modelling}

If we adopt the main three principles of the Web for Semantic Web and create non-unique naming, it creates an environment which will allow Semantic Web to grow like a network effect and become a global phenomenon. But a problem still persists; how to find the right plant within a forest?

Finding the right information whether it is linked or non-linked is of utmost importance. Especially Semantic Web is intended for machines too. Humans have the capacity to reason and analyze the 'right information'. But even for humans it would be time consuming to find the correct information from huge collection of data [4].

Even we human beings create an abstraction of information that we come across and then transform it into knowledge. This process of abstraction and the quality of this process is cornerstone to our understanding of a topic. If we are to instil this sort of mechanism to a machine, it is important that there is a model followed.

Modelling is the process of organizing the information. This solves the problem of finding the 'right information' to a great level of accuracy and further provides: 
- A framework for human communication.

- Meaning for explaining conclusions.

- Structure for mediating various viewpoints.

If the information is categorized and modelled, it is easier to find the right information. This simple principle is the very reason as to why we have markup languages and frameworks like RDF, OWL etc. They provide a mechanism to model the data and provide semantics [9].

\subsection{Resource Description Framework (RDF)}

According to W3C "RDF is a standard model for data interchange on the Web. RDF has features that facilitate data merging even if the underlying schemas differ, and it specifically supports the evolution of schemas over time without requiring all the data consumers to be changed." [11]

- An RDF is generally expressed using something known as a triple which is the most basic unit of information.

- A triple contains a subject, a predicate and an object.

- Namespaces are provided to solve the problem on ambiguity in RDF [12].

A simple instance of an RDF document is shown below:

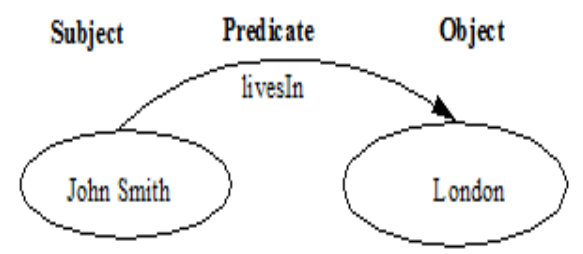

Figure 2: An RDF representation of a fact [13].

The idea of this simple unit of information is that it could be expressed in various formats that could be easily read by machines. The RDFS (RDF Schema) is used for describing the properties and classes of an RDF document. RDFS acts similar to the function of metadata for RDF.

The instance of the triple in the figure is shown in XML and JSON below:

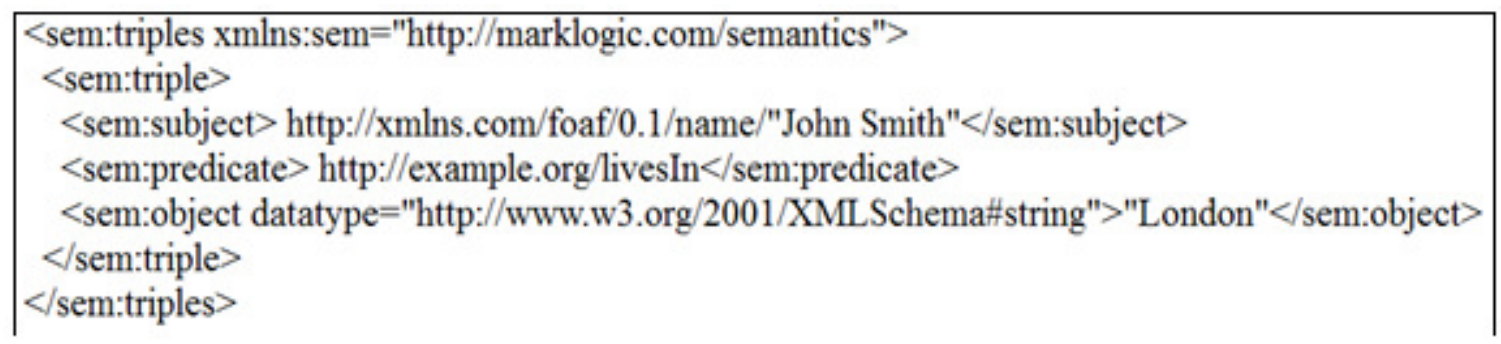

Figure 3: RDF in XML [13]. 


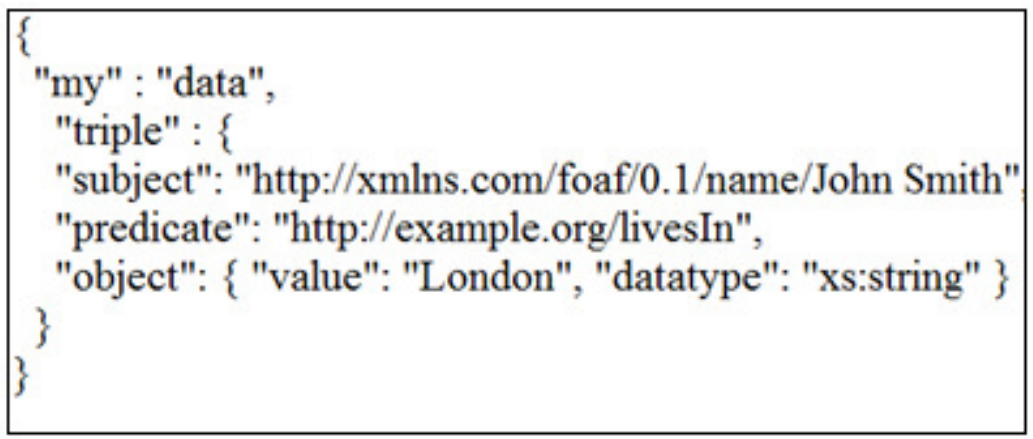

Figure 4: RDF in JSON [13].

\subsection{Web Ontology Language (OWL)}

Web Ontology Language (OWL) is another standard from the W3C Consortium to aid in the progress of Semantic Web.

OWL provides greater machine interpretations by providing additional vocabulary along with other semantics. OWL adds more vocabulary to describe the RDFS (RDF Schema). This concept is the fundamental idea behind the improvement of machine understandability by the use of OWL [14].

OWL was categorized into three sublanguages to fit the need of the users:

OWL Lite - A simple sublanguage that provides classification hierarchy and constraints. The cardinality can only be values of 0 or 1 , thereby restricting and shrinking down the complexities of relations.

OWL DL - The DL stands for 'Description Logics', which is one of the foundational areas for the creation of OWL. OWL DL is for users who wants to achieve the full expressiveness of a topic while ensuring that the computation will finish on a finite set of time.

OWL Full - OWL Full is for users who needs to traverse the entire hierarchy of a subject to its root and even the metadata of the root. It has no computational guarantee as it is quite understandable that this process could be really complex. However, OWL Full pushes to create all the possible meaning of an RDF class [14].

\subsection{Ontology}

An ontology doesn't have a formally accepted definition. However, a vocabulary and ontology is often used with the same meaning. An ontology can be defined as a set of URIs that makes up meaning for a particular topic [15].

The units that make up an ontology would be a set of RDF along with OWL. There are various ontologies that has been created and frequently used. However most of the ontologies are created by humans and machines have little to no say in this matter. 
Examples of few ontologies:

Dublin Core - They have an ontology for metadata of data. Their ontology set includes classes, properties, vocabulary encoding schemes, syntax encoding schemes and collections [16]. All of them have several set of RDFs explaining what the data is about.

Dbpedia - "It is a crowd-sourced community effort to extract structured information from Wikipedia and make this information available on the Web. Dbpedia allows you to ask sophisticated queries against Wikipedia, and to link the different data sets on the Web to Wikipedia data. Furthermore, it might inspire new mechanisms for navigating, linking, and improving the encyclopedia itself." [17]

The above quotation is the official description of Dbpedia from its authors. It would be fair to say that Dbpedia is a Semantic Web version of Wikipedia. The Dbpedia ontology is massive and has 4.2 million instances of objects which include things, person, place, work, organization, and species. This massive amount of data is structured using RDF and OWL. Some of them are linked to other linked-data sources turning Dbpedia into the nucleus of Web of Data as mentioned [18].

There are various other examples of ontologies like FOAF, Good Relations, Music Ontology etc. [15] Now all these ontologies need to be stored somewhere and for that purpose we have Triple Stores.

\subsection{Triple Store}

A Triple Store is a specific kind of database store for storing and retrieving triples. They are stored in the format of subject, predicate and object. For instance, "Alice knows Bob", "Alice is 15 " etc. They are custom built for the purpose of Semantic Web and Linked Data. Similar to any database, the information is retrieved via a query language. A Triple Store has the ability to import and export the required information in RDF format as well [19].

There are a lot of different variants of Triple Stores, some of them are created from scratch and some of them are built on-top of existing SQL and NoSQL databases. Triple Stores are often also called as RDF stores [20].

Few examples of Triple Stores are:

Virtuoso - It is a middleware that supports traditional Relation Database Management Systems (RDBMS) and also has specialized support for RDF document storage and retrieval. It supports multiple protocols and uses a single multi-threaded process. It is also known as Openlink Virtuoso. It provides a SPARQL end point like all the Triple Stores. Virtuoso is well known for its performance in holding huge datasets. For instance, Dbpedia is hosted on a Virtuoso Triple Store [21].

Fuseki - It is a sub project from Apache Jena. It provides an RDF server that can be a Triple Store, which can be administered and managed via REST protocols. It can run as a service on a remote machine, a WAR (Java Web application file) or as a standalone server. Fuseki supports 
SPARQL 1.1 and also has added in logging support to keep a close watch of what happens on the triple store.

Fuseki's latest version v2, provides security through Apache Shiro. It adds cryptography and session management to Fuseki [22].

These Triple Stores have an endpoint for SPARQL to query the RDF documents.

There has been a lot of study conducted to find the most optimal Triple Stores. A comparison done between Apache Fuseki, Blazegraph, Sesame and Virtuoso is shown in Table 2.

Table 1: Comparison of Triple Stores [26]

\begin{tabular}{|l|l|l|l|}
\hline Name & License & Deployment & Language \\
\hline Apache Fuseki & Apache License 2 & Standalone or WAR & Java \\
\hline Blazegraph & GPLv2 or commercial & Standalone or WAR & Java \\
\hline Sesame & BSD & WAR & Java \\
\hline Virtuoso & GPL & Native & C \\
\hline
\end{tabular}

A performance benchmarking was done by Vladimir Mironov et al. of various Triple Stores. The findings were also a positive addition to the selection of Fuseki [27].

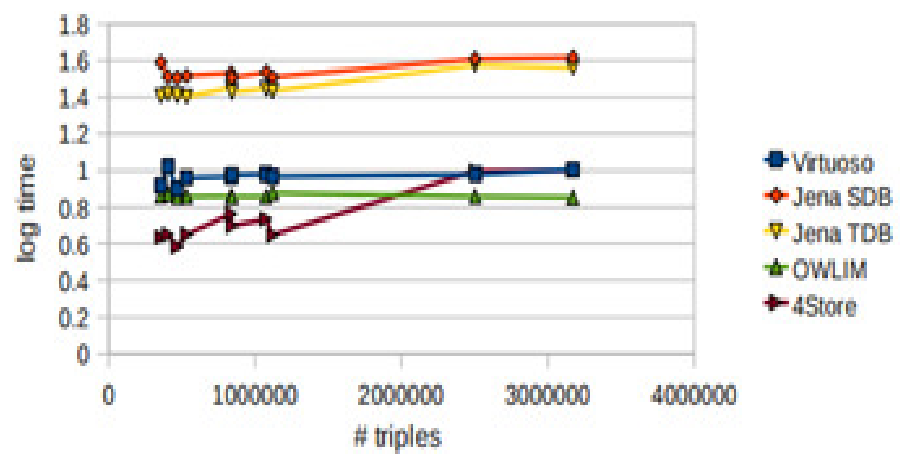

Figure 5: Average Response Time of various Triple Stores [27]

Apache Fuseki is based on Jena TDB and currently only known as Fuseki. So it is evident from the conclusion of the study (See Figure 8), that Fuseki is a winner in case of average response time of queries.

\subsection{SPARQL}

SPARQL is another W3C standard in the category for Semantic Web. It is a query language similar to that of Structured Query Language (SQL) for Relational Database Management Systems (RDBMS). SPARQL is used to query RDF documents. RDF documents as explained in 
Section 2.2.1, are depicted in the format of labelled triples. This allows a graph representation of the RDF document. So SPARQL queries can be result sets of graphs [23].

The results set is either a literal (value) or a URI. The ability to fetch the literal or even convert URI into their labels provide a direct and easy way for applications to use the result set directly.

Table 2: Keywords used by SPARQL [23].

\begin{tabular}{|l|l|l|l|l|l|l|}
\hline BASE & SELECT & ORDER BY & FROM & GRAPH & STR & isURI \\
\hline PREFIX & CONSTRUCT & LIMIT & FROM NAMED & OPTIONAL & LANG & isIRI \\
\hline & DESCRIBE & OFFSET & WHERE & UNION & LANGMATCHES & isLITERAL \\
\hline & ASK & DISTINCT & & FILTER & DATATYPE & REGEX \\
\hline & & REDUCED & & a & BOUND & true \\
\hline & & & & & sameTERM & false \\
\hline
\end{tabular}

Table 2, shows the list of most commonly used keywords used by SPARQL. A SPARQL Abstract Query is a tuple (E, DS, QF) where:

- $\mathrm{E}$ is a SPARQL algebra expression

- DS is an RDF Dataset

- $\mathrm{QF}$ is a query form

Every triple store has a SPARQL endpoint as mentioned. But triple stores generally can restrict the kind of SPARQL queries than can be executed. So permissions can be controlled at the application level.

\section{DISCUSSIONS}

We start our discussion with two main views on Semantic Web by different authors on this topic. These approaches can be collated in to two different sets of a Venn Diagram namely, Semantic and Web. The main driver in semantic is artificial intelligence and in Web is Smart Linked Data.

\section{Semantic Approach}

As discussed earlier, when Tim Berners Lee spoke about having personal agents, it meant that these software agents would be able to interpret data and accomplish personal task for us. The way this would be done would be with the concept of inference or reasoning. This is a very common approaches in Knowledge based AI where new facts are inferred from existing facts and reasoning can be done on existing datasets. This would give the ability to agents to not only use the information that has been given to them at the start but also create new intelligence on their own.

A survey was done by Jorge Cordoso titled "The Semantic Web Vision: Where are We?", which has a criterion that is quite interesting to our paper [31]. They list out the top reasons as to why Semantic Web is in use in Figure 5, and sharing common understanding of information structure among people and agents are on top. 


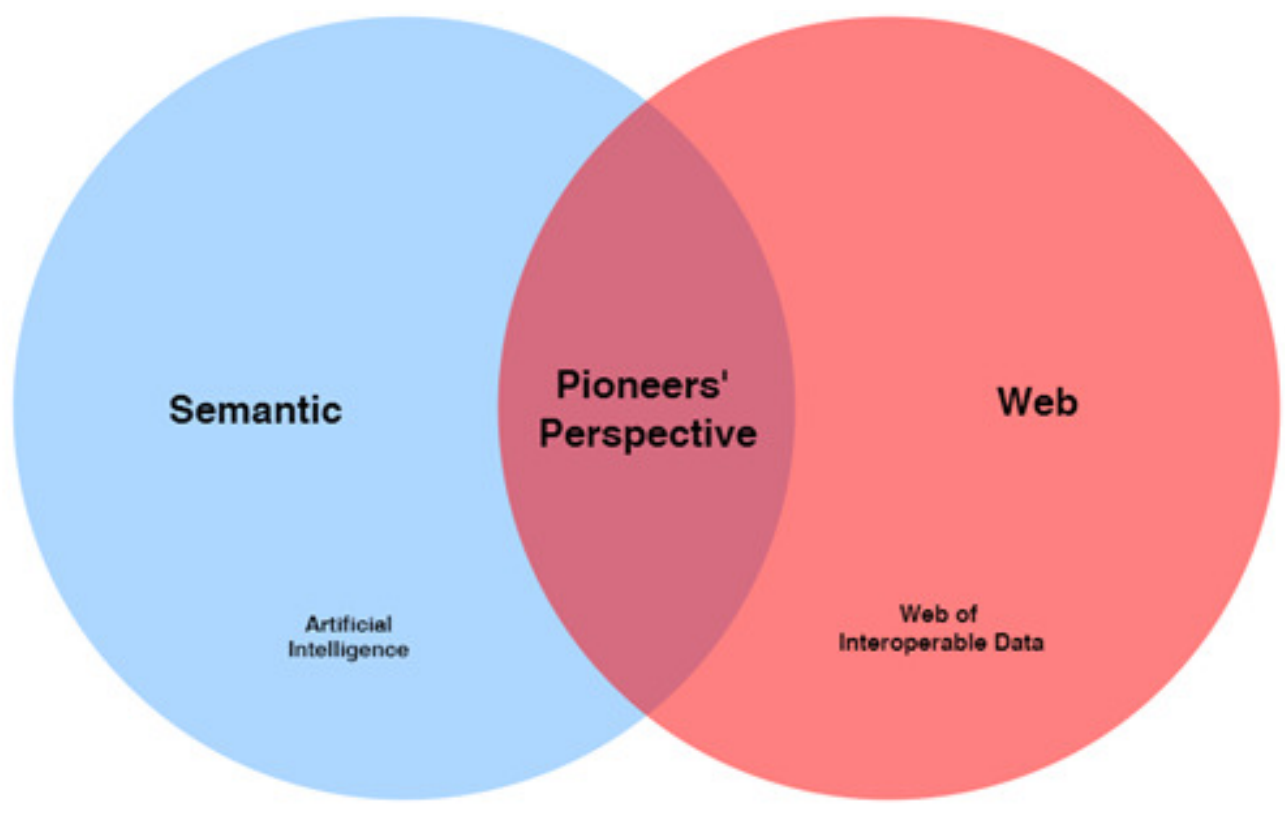

Figure 6: The Pioneers' Perspective

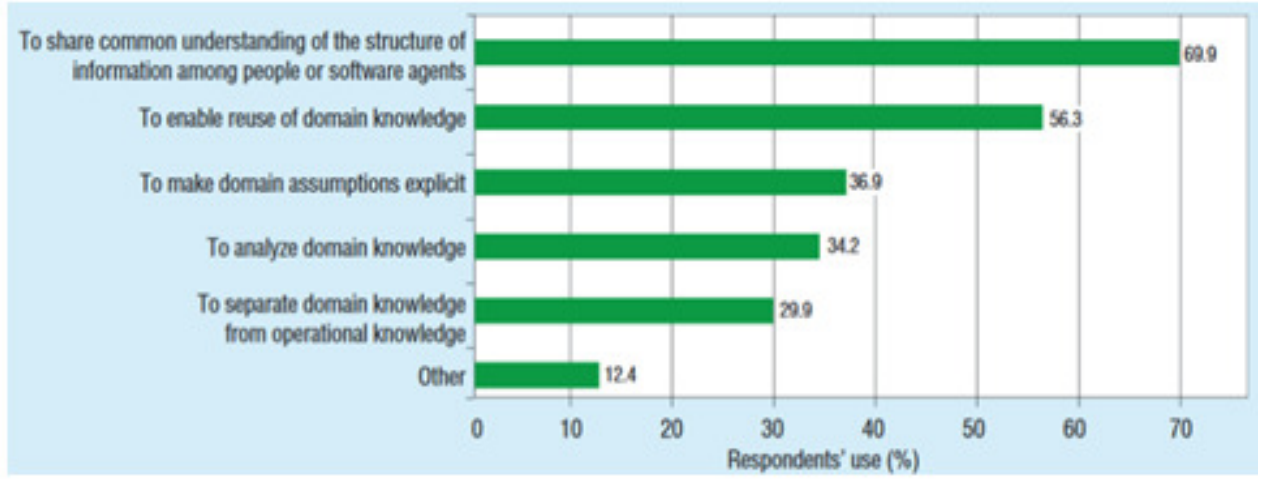

Figure 7: Reason of Semantic Web usage [31].

Roughly about $70 \%$ of the users find that Semantic Web's main purpose is to share common understanding of the structure of information. This enhances the machines to reason and infer on the same base knowledge.

The supporters of this approach were interested in the semantic aspects rather than the hyperlinking of resources aspect. In a nutshell, here semantic web is used for performing artificial intelligence research and developing practical solutions.

\section{Web Approach}

In this approach, the Linked Data is the driving force. Here the usage of Semantic Web has been to connect data available at different sources. The source could be structured or unstructured giving rise to a flexible model of data usage. For instance, a crowd sourced project called as The Linked Open Data (LOD) has 31+ billion facts in the LOD cloud as of 2014. 


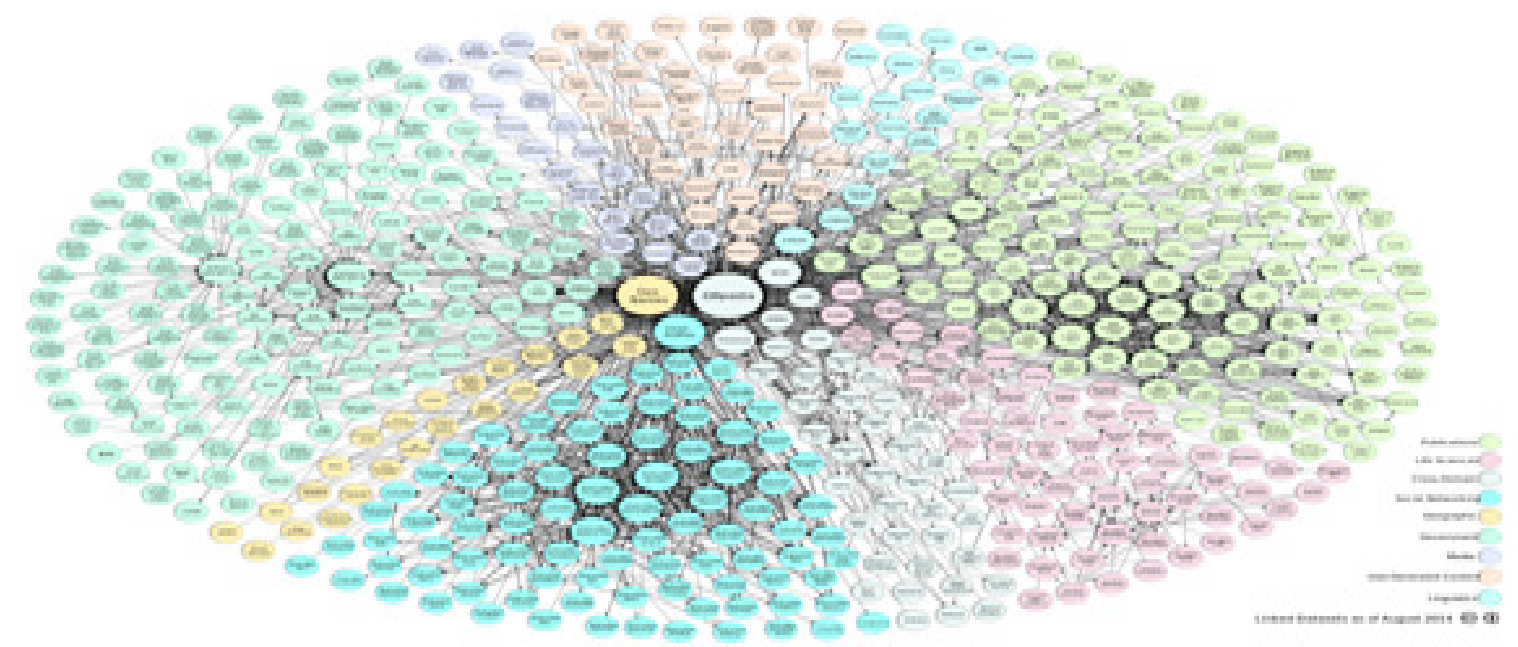

Figure 8 : Linked Open Data Cloud [24]

The supporters of this approach believe the Artificial intelligence is an unwanted liability as they perceive their application would be become more complex.

\section{Semantic and Web Intersection: The Pioneers Approach}

In this approach, the pioneers' envisioned the use of Semantic Web where, inferencing is a major aspect. Inferencing and reasoning would essentially lead to knowledge management. This comes from the first part of the semantic approach. But the knowledge that is derived from linked data is more insightful as the data is processed from various sources. And this is based on the second approach, which is the Web approach. But there are quite few concerns shown by the skeptics mainly about the usage of such a system. This is being asked over and over again [25].

James Hendler along with Sir Tim Berners-Lee, responds to this in his presentation by arguing that once we have enough semantic data everyone would want to become a Semantic Web user including governments. The open data project which more and more governments are joining is a clear indication towards this phenomenon [8].

Sir Tim Berners-Lee in his famous TED talk says "The power to ask questions, questions that bridge across different disciplines is a complete sea change." [4]

This is done through Semantic Web. The relationship among various things form the 'bridges' that Sir Tim Berners-Lee speaks about. This is the very basis of enabling cross discipline analysis and research.

James Hendler talks about the application of Semantic Web and how pervasive it has become. He provides enough examples to prove that knowingly or unknowingly everyone uses the fruits of Semantic Web. Facebook's open graph protocol, Oracle's Semantic Web extensions, Google's search result etc. are all ways in which everyone is already a user of Semantic Web [10].

As discussed above we find that these approaches are not mutually exclusive but in practice this is what the pioneers approach actually should be. 


\section{CONCLUSION}

After a brief analysis of the Literature Review in Semantic Web, we are able to understand the vision of the pioneers of Semantic Web. The main target was to transform the current Web to that which has smarter data. This would in turn allow the machines to understand and use the data better.

We have broken down Semantic Web into two approaches and explained them individually to reach to an intersection. Semantic Web is a technology that has great potential for the betterment of the society. But as explained, the focus of researchers is usually only on one approach. And the real value of Semantic Web is at its intersection between the two approaches - The Pioneers' Perspective.

\section{REFERENCES}

[1] S. T. Berners-Lee, 2016. [Online]. Available: https://www.w3.org/standards/webdesign/accessibility. [Accessed 05 January 2016].

[2] F. Heylighen and J. Bollen, "The World-Wide Web as a Super-Brain: from metaphor to model," Cybernetics and Systems '96, vol. R. Trappl (ed.), 1996.

[3] C. ROBERTS, 2013. [Online]. Available: http://www.technologybloggers.org/science/how-manyhuman-brains-would-it-take-to-store-the-internet/. [Accessed 08 January 2016].

[4] S. T. Berners-Lee, "The next Web," TED Talks

(https://www.ted.com/talks/tim_berners_lee_on_the_next_web?language=en), 2009.

[5] M. S. GUHA R, "Schema.org: Evolution of Structured Data on the Web," Communications Of The ACM [serial on the Internet], pp. 44-51, 2016.

[6] P. A. Halevy, "The Unreasonable Effectiveness of Data," IEEE Intelligent Systems, vol. 24, no. 2, pp. 8-12, 2009.

[7] statista.com, "Internet of Things (IoT): number of connected devices worldwide from 2012 to 2020 (in billions)," 2016. [Online]. Available: http://www.statista.com/statistics/471264/iot-number-ofconnected-devices-worldwide/. [Accessed 20 February 2016].

[8] J. Hendler, Writer, "Why the Semantic Web will Never Work". [Performance]. Keynote, European Semantic Web Conference, Heraklion, June, 2011.

[9] J. Hendler and D. Allemang, Semantic Web for the Working Ontologist, 2nd ed., Elsevier Inc., 2011.

[10] J. Hendler, "Semantic Web: The Insider Story," 2014. [Online]. Available: http://www.slideshare.net/jahendler/semantic-web-the-inside-story. [Accessed 15 March 2016].

[11] W3C, 2016. [Online]. Available: https://www.w3.org/RDF/. [Accessed 15 January 2016].

[12] B. DuCharme, Learning SPARQL, O'Reilly Media Inc., 2013. 
[13] M. Community, 2016. [Online]. Available:

https://docs.marklogic.com/media/apidoc/8.0/guide/semantics/intro/intro-2.gif. [Accessed 20 January 2016].

[14] W. Consortium, 2004. [Online]. Available: https://www.w3.org/TR/2004/REC-owl-features20040210/\#s1.2. [Accessed 20 January 2016].

[15] Semanticweb.org, 2012. [Online]. Available: http://semanticweb.org/wiki/Ontology. [Accessed 25 January 2016].

[16] D. C. M. Registry, "Browse the registry by classification type," 2016. [Online]. Available: http://dcmi.kc.tsukuba.ac.jp/dcregistry/navigateServlet. [Accessed 20 March 2016].

[17] "Dbpedia - About," Dbpedia.org, 2016. [Online]. Available: http://dbpedia.org/about. [Accessed 15 March 2016].

[18] Dbpedia.org, "Dbpedia.org I Nucleus for the Web of Data," 2016. [Online]. Available: http://wiki.dbpedia.org/use-cases/nucleus-web-data. [Accessed 26 February 2016].

[19] J. Rusher, "Triple Store," 2016. [Online]. Available: https://www.w3.org/2001/sw/Europe/events/20031113-storage/positions/rusher.html. [Accessed 27 February 2016].

[20] K. Cagle, "Semantics + Search : MarkLogic 7 Gets RDF," 22 December 2013. [Online]. Available: http://blogs.avalonconsult.com/blog/search/semantics-search-marklogic-7-gets-rdf/. [Accessed 27 February 2016].

[21] W3C, "OpenLink Virtuoso," 11 February 2012. [Online]. Available:

https://www.w3.org/2001/sw/wiki/OpenLink_Virtuoso. [Accessed 18 March 2016].

[22] jena.apache.org, "Apache Jena Fuseki," 2015. [Online]. Available: https://jena.apache.org/documentation/fuseki2/index.html. [Accessed 10 March 2016].

[23] W3C, "SPARQL Query Language for RDF," 2008. [Online]. Available: https://www.w3.org/TR/rdfsparql-query/. [Accessed 27 February 2016].

[24] U. o. Manheim, "State of the LOD Cloud 2014," 30 August 2014. [Online]. Available: http://linkeddatacatalog.dws.informatik.uni-mannheim.de/state/. [Accessed 06 March 2016].

[25] M. Bernstein, "MIT CSAIL Research," October 2009. [Online]. Available: http://haystack.csail.mit.edu/blog/2009/10/25/tales-of-a-semantic-web-skeptic/. [Accessed 5 March 2016].

[26] utecht.github.io, "Comparing Triple Stores," UTecht Blog, 2015. [Online]. Available: http://utecht.github.io/comparing-triplestores/. [Accessed 12 March 2016].

[27] V. Mironov, N. Seethappan, W. Blondé, E. Antezana, B. Lindi and M. Kuiper, "Benchmarking triple stores with biological data," Proceedings of the 3rd International Workshop on Semantic Web Applications and Tools for the Life Sciences, December, 2010.

[28] V. Janev and S. Vranes, "Semantic Web Technologies: Ready for Adoption?," IT Professional, vol. 11 , no. 5, 2009. 
[29] G. Raf, "Tracing the origins of the semantic web," Journal of the American Society for Information Science and Technology, vol. 64, no. 10, pp. 2173-2181, 2013.

[30] S. M. Benslimane, M. Malki and A. Lehirech, "Towards ontology-based semantic web from dataintensive web: A reverse engineering approach," IEEE International Conference on Computer Systems and Applications, pp. 771-778, 2006.

[31] J. Cordoso, "The Semantic Web Vision: Where Are We?," IEEE Intelligent Systems, vol. 22, no. 5, 2006.

\section{AUTHORS}

Salih Ismail received bachelors (hons) degree in Information Technology from University of Bedfordshire. He is currently completed in MSc in Computer Systems Management from Heriot Watt University. $\mathrm{He}$ has worked on finding commonalities between Semantic entities. His research areas of interest include Network Security, Semantic Web, Internet of Things etc. He has been running an IT company in Dubai.

Talal Shaikh is currently Assistant Professor at Heriot Watt University Dubai in the School of Mathematics and Computer Science (MACS). Some of the subjects he teaches are Network Applications, Software Engineering and Artificial Intelligence. His research area of interest is making "things" such as robots and devices "talk" to one another using software in order to carry out a task and achieve a common goal. His ongoing research covers ubiquitous and pervasive computing, Machine to Machine (M2M) technologies and the Internet of Things. His academic area of

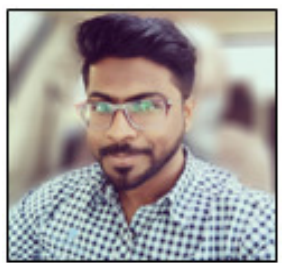
interest covers student learning and student experience. 